\title{
Far-infrared spectroscopy of nanoscopic InAs rings
}

\author{
Agustí Emperador, Martí Pi, and Manuel Barranco \\ Departament ECM, Facultat de Física, Universitat de Barcelona, E-08028 Barcelona, Spain \\ Axel Lorke \\ Sektion Physik, LMU München-Geschwister-Scholl-Platz 1, D-80539 München, Germany
}

(Received 28 October 1999; revised manuscript received 2 March 2000)

\begin{abstract}
We have employed time-dependent local-spin-density theory to analyze the far-infrared transmission spectrum of InAs self-assembled nanoscopic rings recently reported [A. Lorke et al., Phys. Rev. Lett. (to be published)]. The overall agreement between theory and experiment is fairly good, which on the one hand confirms that the experimental peaks indeed reflect the ringlike structure of the sample, and on the other hand, asseses the suitability of the theoretical method to describe such nanostructures. The addition energies of oneand two-electron rings are also reported and compared with the corresponding capacitance spectra.
\end{abstract}

Recent progress in nanofabrication techniques ${ }^{1,2}$ has allowed us to construct self-assembled nanoscopic InGaAs quantum rings occupied with one or two electrons each, and submitted to perpendicular magnetic fields $(B)$ of up to $12 \mathrm{~T}$. These are spectroscopic data available on rings in the scatterfree, few electrons limit in which quantum effects are best manifested. Previous spectroscopic studies dealt with microscopic rings ${ }^{3}$ in $\mathrm{GaAs}-\mathrm{Ga}_{x} \mathrm{Al}_{1-x} \mathrm{As}$ heterostructures, fairly well reproduced by classical or hydrodynamical models. ${ }^{4,5}$

In spite of the lacking of experimental information, the study of nanoscopic ring structures has already attracted a strong theoretical interest. ${ }^{6-11} \mathrm{We}$ recall that due to the nonapplicability of the generalized Kohn theorem, a very rich spectroscopic structure is expected to appear in few electrons nanorings, as anticipated by Halonen, Pietiläinen, and Chakraborty ${ }^{9}$ and also found in recent works. ${ }^{12,13}$

In this paper we attempt a quantitative description of some spectroscopic and ground-state $(g s)$ properties of the experimentally studied nanoscopic rings ${ }^{1,2}$ using currentdensity (CDFT) and time-dependent local-spin-density (TDLSDFT) functional theories. The reason for such an attempt is twofold: on the one hand, to contribute to put on a firm basis the interpretation of current experiments as manifestation of actual properties of few-electrons ring-shaped nanostructures; on the other hand, although in principle exact results could be obtained for one and two electron rings using, e.g., exact-diagonalization methods, ${ }^{8,9}$ the present one can also be applied to many electron systems, ${ }^{12}$ and we think it is worthwhile to explore the capabilities and limitations of density-functional methods to describe such small rings.

Following Ref. 6, we have modeled the ring confining potential by a parabola,

$$
V^{+}(r)=\frac{1}{2} m \omega_{0}^{2}\left(r-R_{0}\right)^{2}
$$

with $^{2} R_{0}=14 \mathrm{~nm}$ and the frequency $\omega_{0}$ fixed to reproduce the high-energy peak found in the far-infrared (FIR) transmission spectrum at $B=0$. For $N=2$ electrons this yields $\omega_{0} \sim 12.3 \mathrm{meV}$. The electron effective mass $m^{*}=0.063$ (we write $m=m^{*} m_{e}$ with $m_{e}$ being the physical electron mass) and effective gyromagnetic factor $g^{*}=-0.43$ have been taken from the experiments, ${ }^{14-16}$ and the value of the dielectric constant has been taken to be $\epsilon=12.4$.

To obtain the structure of the GS we have resorted to CDFT as described in Refs. 17 and 18, and to obtain the FIR absorption we have used TDLSDFT as described in Ref. 19, which has been recently applied to the ring geometry. ${ }^{12}$ It is worthwhile to point out that we have not found any significant difference between using CDFT or LSDFT to describe the GS of the studied rings in the range of $B$ values of the present work. The suitability of CDFT to study small electronic system has been shown by Ferconi and Vignale ${ }^{17}$ comparing the results obtained for a dot with $N=2$ electrons with exact and Hartree-Fock calculations. For larger electron numbers the method has also been successfully tested against diffusion Monte Carlo calculations. ${ }^{20}$ Although these comparisons refer to quantum dotlike confining potentials, one should not expect that the situation drastically changes when using other smooth confining potentials as that of Eq. (1). Indeed, the absorption energies and intensities that we report in the following are qualitatively similar to those obtained by Halonen, Pietiläinen, and Chakraborty ${ }^{9}$ for quasi-onedimensional rings from an exact-diagonalization method. We refer the reader to Refs. 18 and 19 for a comprehensive exposure of CDFT and TDLSDFT, of direct applicability here only changing the shape of the confining potential from a dotlike one to a ringlike one.

The results obtained for the $N=2$ ring are presented in Figs. $1-4$. We have used a small temperature $T=0.1 \mathrm{~K}$ to work them out. Figure 1 shows that the ring becomes polarized near $B=3$ T. Besides, two other $B$-induced changes arise in the GS at $B \sim 8 \mathrm{~T}$ and, more weakly, at $B \sim 14 \mathrm{~T}$. These changes can be traced back to single-particle (SP) level crossings, ${ }^{2}$ and are a distinct feature of few electron rings that make their capacitance spectrum and FIR absorption qualitatively different from these of dots. Others, as the existence of $\Delta n=1$ and $\Delta n=0$ transitions, are common to rings and dots as well, since they are not specific of a ring confining geometry but of the breaking down of the generalized Kohn theorem when the confining potential is not a harmonic oscillator centered at $r=0$. 


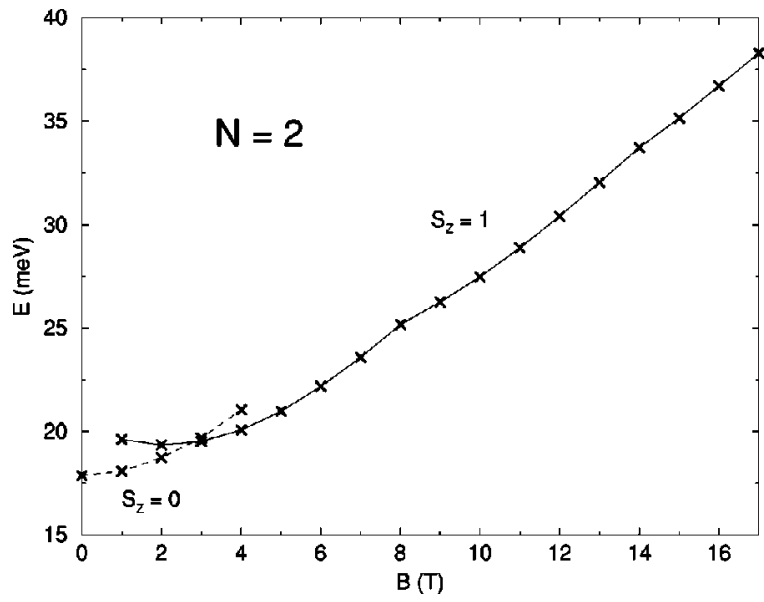

FIG. 1. Total energy of the $N=2$ ring as a function of $B$. The dashed line corresponds to an $S_{z}=0 \mathrm{GS}$, and the solid line to an $S_{z}=1 \mathrm{GS}$. The ring becomes fully polarized near $B=3 \mathrm{~T}$.

As displayed in Fig. 2, the changes in the $B$ slope appear when an occupied SP level is substituted by an empty one. At $B \sim 8 \mathrm{~T}$, this involves the substitution of the $l=0$ SP level by the $l=2$ one, and at $B \sim 14 \mathrm{~T}$ the $l=1 \mathrm{SP}$ level is substituted by the $l=3$ one. ${ }^{21}$ Other level crossings do not involve such substitutions, but a different ordering of the occupied levels and do not seem to produce a substantial effect (see for instance the crossings at $B \sim 6$ and $\sim 11.5 \mathrm{~T}$ ).

The experimentally observed change in the FIR spectrum around $B=8 \mathrm{~T}$ has been attributed ${ }^{2}$ to the crossing of $l=0$ and 1 SP levels on the basis of a simple single-electron model (see also Fig. 5). A realistic description of the crossings requires to incorporate in the theoretical description the spin degree of freedom, of which single electron or Hartree models ${ }^{13}$ lack whereas CDFT or LSDFT do not. Yet, we confirm the finding ${ }^{2}$ that a magnetic induced transition takes place in the GS when approximately 1 flux quantum penetrates the effective interior area of the ring at $B \sim 8 \mathrm{~T}$, and predict another one at $B \sim 14 \mathrm{~T}$ when this area is penetrated by $\sim 2$ flux quanta.

The changes in the $B$ slope of the total energy correlate well with these in the electronic chemical potential (the en-

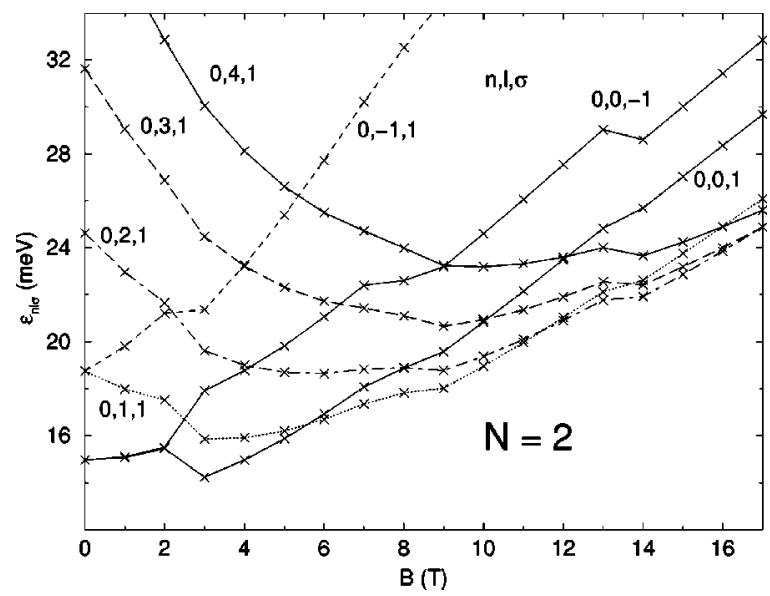

FIG. 2. Several $n=0$ SP energies for the $N=2$ ring as a function of $B$. The quantum labels $(n, l, \sigma)$ of the SP states are also indicated.

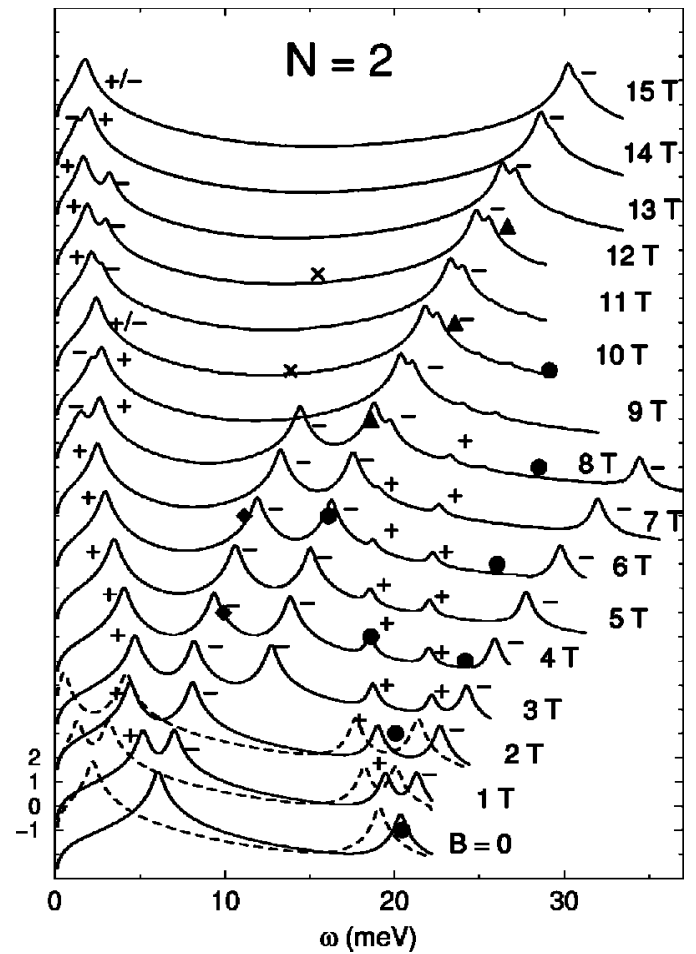

FIG. 3. FIR absorption vs excitation energy (solid lines) for $N$ $=2$ and $B=0$ to $15 \mathrm{~T}$. The curves have been offset for clarity, and are drawn in an arbitrary logarithmic scale common to all $B$ values. To allow for a comparison of the relative intensity of the peaks corresponding to a given $B$, we have indicated the scale values of the $B=0$ spectrum. The symbols represent the experimental peak energies (Ref. 2). The dashed lines at $B=0,1$, and $2 \mathrm{~T}$ represent the dipole spin modes.

ergy of the last occupied SP level in Fig. 2). The gross structure of the chemical potential and total energy displays the well known periodic, Aharonov-Bohm-type oscillation found in extreme quasi-one-dimensional SP models: $:^{2,6,10}$

$$
\epsilon_{l}=\frac{\hbar^{2}}{2 m R_{0}^{2}}\left(l-\frac{e}{\hbar c} R_{0}^{2} B\right)^{2}=\frac{\hbar^{2}}{2 m R_{0}^{2}}\left(l-\frac{\Phi}{\Phi_{0}}\right)^{2},
$$

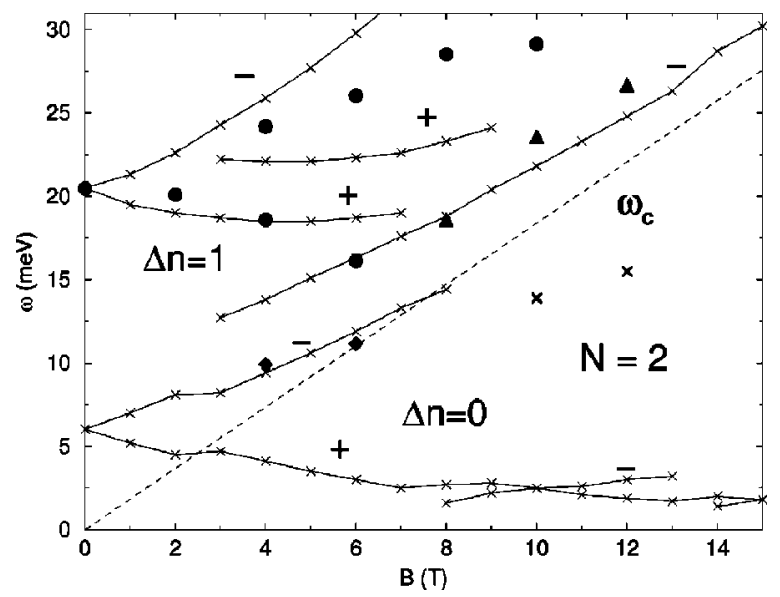

FIG. 4. Energy of the more intense CDE's as a function of $B$ for $N=2$. The dashed line represents the cyclotron frequency $\omega_{c}$, and the solid lines are drawn to guide the eye. The thick symbols represent the experimental data (Ref. 2). 


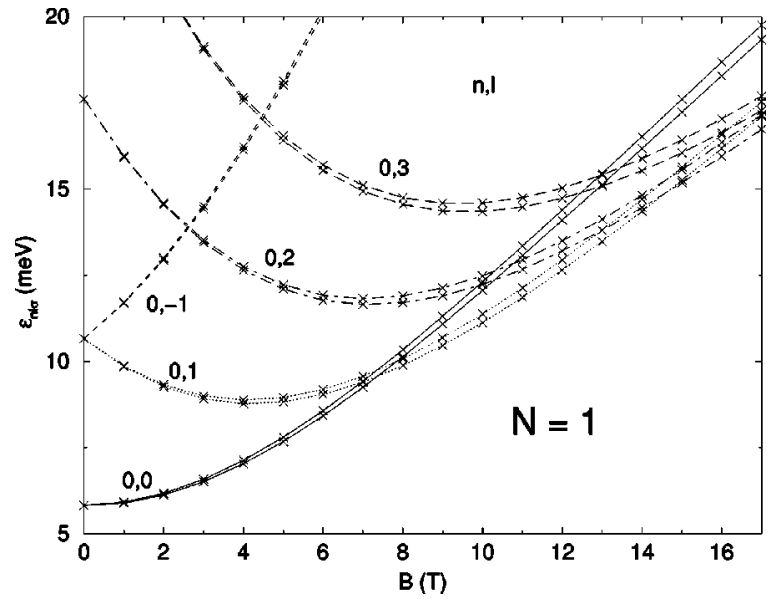

FIG. 5. Several $n=0 \mathrm{SP}$ energies for the $N=1$ ring as a function of $B$. The lower energy state of each $(n, l)$ pair has spin up.

where $\Phi=\pi R_{0}^{2} B$ and $\Phi_{0}=h c / e$. Yet, the experimental ring is far from being quasi-one-dimensional. As a consequence, and in agreement with experiment, Figs. 2 and 5 show that the crossing between the $l=0$ and 1 SP levels occurs at $\Phi$ $\sim \Phi_{0}$ instead of at $\Phi_{0} / 2$, as it would be if Eq. (2) holds.

The experimental FIR resonances have been grouped $^{2}$ into different modes using a different symbol for each group. Here, we have used the same symbol to represent the experimental resonances in Figs. 3 and 4. Figure 3 shows the FIR absorption in an arbitrary logarithmic scale as a function of the excitation energy. The curves have been offset for clarity. Charge-density excitations (CDE) can be identified as "ridges" in the plot, allowing to make a sensible comparison with experiment not only of the peak energies themselves, but also of the way the experimental modes have been grouped. A plot of the more intense CDE's is presented in Fig. 4 as a function of $B$, which is qualitatively similar to that of Halonen, Pietiläinen, and Chakraborty ${ }^{9}$ for an $N=2$ quantum dot with a repulsive Gaussian impurity in its center.

It is worthwhile to recall that the results shown in Refs. 9 and 10 refer to a system which is close to an ideal onedimensional ring. These kinds of configurations sustain interesting effects, such as fractional Aharonov-Bohm oscillations, i.e., the decrease of period and amplitude of oscillations of the GS energy ${ }^{10}$ as a function of $\Phi / \Phi_{0}$, which we have not found in the rather broad rings representing those experimentally studied. ${ }^{1,2}$ Although it is not the point of the present work, we have tried to elucidate whether CDFT could address the fractional oscillations. To make the ring more quasi-one-dimensional, we have increased the confinement taking a frequency $\omega_{0}$ three times larger. For this configuration we have indeed found fractional oscillations superposed to the steadily increasing of $E(B)$ as shown in Fig. 1. As thoroughly discussed in Ref. 10, these oscillations are caused by the exchange Coulomb energy which favors the binding of the triplet state that comes down in energy with respect to the others.

For completeness, we also show in Fig. 3 the spin dipole modes $^{19}$ for the $B$ values such that the ring is not fully polarized. The observed shifts between spin and charge modes are a measure of the importance of the electron-electron interaction, which affects more the low-energy than the highenergy peaks.
Figures 3 and 4 show that the FIR absorption is split into two large groups of peaks. The low-energy peaks correspond to transitions involving only $n=0 \mathrm{SP}$ levels and are $\Delta n=0$ transitions, whereas the high-energy peaks involve $n=0$ and 1 SP levels and are $\Delta n=1$ transitions. One can easily distinguish two sets of resonances, a low-lying $\Delta n=0$ one, and a high-lying $\Delta n=1$ one exhibiting the usual Zeeman splitting when a magnetic field is applied. The intensity of the high-energy resonance is more than one order of magnitude smaller than that of the low-energy one. Experimentally, both sets have similar oscillatory strengths, whereas TDLSDFT yields a $\sim 90-10 \%$ share at most. The calculations in Ref. 9 also yield rather different absorption intensities to these resonances. We have checked that the computed spectrum fulfills the $f$-sum rule ${ }^{19}$ to within $\sim 98 \%$, thus leaving no room for higher energy, $\Delta n>1$ peaks to appear within TDLSDFT.

Besides these Zeeman-split resonances, several others show up in the spectrum. We have identified with a $+(-)$ sign these involving changes $\Delta|L|=1(-1)$ in the total orbital angular momentum ${ }^{21}$ with respect to that of the GS.

At $B \sim 8 \mathrm{~T}$, the positive $B$-dispersion brach of the $\Delta n=0$ resonance disappears, and a very low-lying, positive $B$-dispersion branch shows up. The origin of this transition is the magnetic-induced change in the GS, as it can be easily inferred looking at the $n=0$ SP levels plotted in Fig. 2 and using the dipole selection rule to identify the ones involved in the non-spin-flip excitation. A similar transition occurs at $B \sim 14 \mathrm{~T}$. They are the microscopic explanation of the appearance and disappearance of the ridges shown in Fig. 3, also found for few electron nanoscopic rings. ${ }^{9,12}$ It is worthwhile to notice that the rich structure appearing in these rings (see below the $N=1$ case) is a peculiarity that has its origin in the smallness of $N$. When $N$ is just a few tens, many electron-hole pairs contribute to the building of the resonances and no drastic changes appear in the FIR absorption. $^{12}$ Yet, another clear signature which distinguishes a ring from a dot configuration appears for a number of electrons as small as $N=5$, namely, the existence of two edge modes, one with cyclotronlike polarization and another with anticyclotronlike polarization ${ }^{3,12}$ which arise due to the existence of an inner and an outer ring edge.

The more conspicuous disagreement between theory and experiment is in the "cross" modes at $B=10$ and $12 \mathrm{~T}$ (see also Fig. 6). A possible explanation ${ }^{2}$ is that these resonances originate from dots that have not developed into rings in the fabric process, thus not experiencing a change in their GS at $B \sim 8 \mathrm{~T}$. This change is a crucial signature that distinguish the ring from the dot configuration: we have checked that for a two electron dot and realistic confining potentials (parabolic and jellium disk), only SP states with $l=0$ and $l=1$ are occupied depending on the $B$ value, and these SP levels do not cross, whereas we have seen they do for a ring. This causes the observed structure in the capacitance spectrum at $B \sim 8 \mathrm{~T}$, and the changes in the FIR absortion at the same $B$ value.

As mentioned in Ref. 2, experimental data are also available for the case of one electron per ring, $N=1$. The gate voltage tunability of the samples allows for a direct comparison between the $N=1$ and the $N=2$ case, since both were taken on the same sample during the same cooldown. For the 


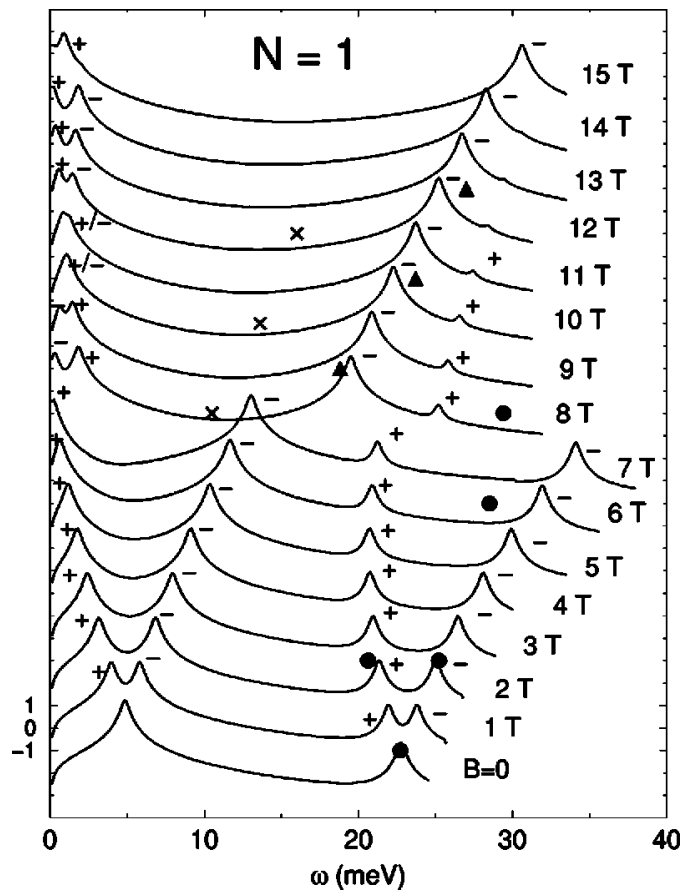

FIG. 6. Same as Fig. 3 for $N=1$. The experimental data are from Ref. 22.

calculations, we followed the same procedure as in the $N$ $=2$ case and fixed the $\omega_{0}$ as to reproduce the high-energy resonance at $B=0$. This yields $\omega_{0} \approx 13.5 \mathrm{meV}$.

Figure 5 shows several $n=0$ SP levels of the $N=1$ ring. For this system, the total energy $E(1)$ is simply the energy of the lowest SP level, $E(1)=\mu(1)$. This has been used to calculate the addition energy $\mu(2)=E(2)-E(1)$ shown in Fig. 8. The FIR absorption and the energy of the more intense $N=1$ CDE's are plotted in Figs. 6 and 7 as a function of $B$.

We thus see that the experimental data on FIR transmission spectroscopy reflects that the surface ring morphology of the experimental samples has indeed being translated to a true underlying electronic ring structure, ${ }^{2}$ and that a fair agreement can be found between TDLSDFT calculations and experiment. Our calculations also give support to the way the experimental resonances have been grouped, with the only

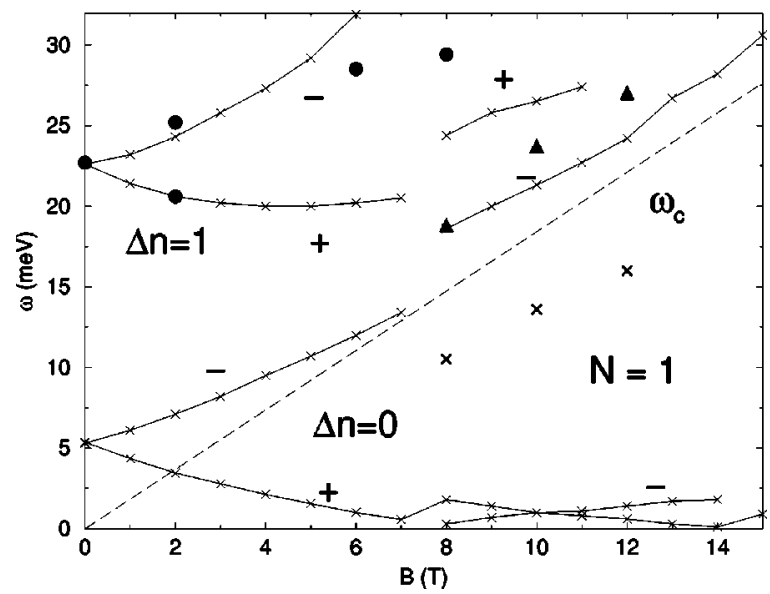

FIG. 7. Same as Fig. 4 for $N=1$. The experimental data are from Ref. 22.

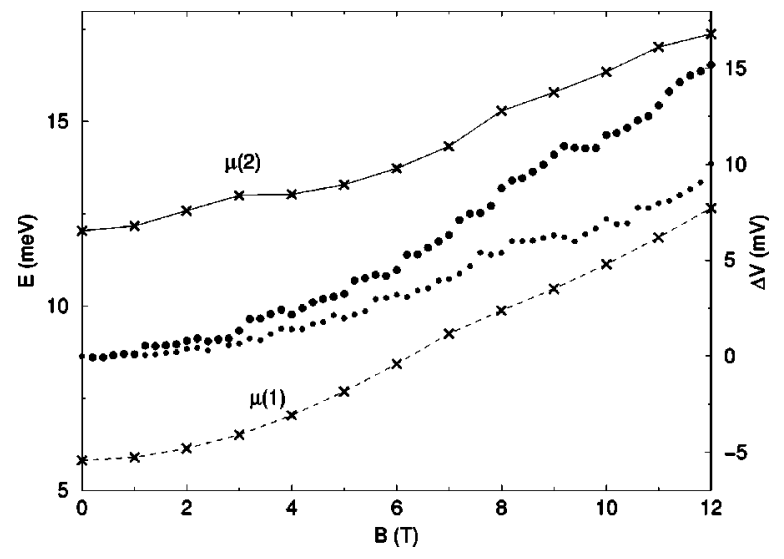

FIG. 8. Addition energies as a function of $B$ (left vertical scale). The symbols are the experimental capacitance data (Refs. 2 and 22) (right vertical scale). Large dots correspond to $N=2$, and small dots to $N=1$.

doubt of the "dot" peak at $B=6 \mathrm{~T}$ and $\omega \sim 16.1 \mathrm{meV}$ which could also be a "triangle" peak of $(-)$ character because in this region both branches merge. To unambiguously arrange the peaks into branches and disentangle the $B$ dispersion of the modes, it would be essential to experimentally assign the polarization state to the main CDE's, as is has been done for antidot arrays. ${ }^{23}$ This is crucial in the analysis of the theoretical FIR absorption, which otherwise does not allow us to distinguish between peak fragmentation and different plasmon branches in some cases.

Besides the "cross" peaks already discussed, another shortcoming in the calculation is a clear overestimation of the peak energy of the ( - ) high energy $\Delta n=1$ mode, which also lacks of some strength. These drawbacks are also in the calculations of Ref. 9. It is alike that using other simple confining potentials, like a jellium ring ${ }^{12}$ or that of Ref. 11 which yields analytical SP wave functions in the noninteracting case, would improve the agreement. Using more complex potentials could help, at the undesidered cost of introducing additional adjustable parameters and obscuring the main physics in these many-parameter models. Furthermore, there are other possible sources of uncertainty, as for example the precise value of the ring radius $R_{0}$ (we have taken that of Ref. 2, but larger values would also be acceptable), and the values of $m^{*}, g^{*}$, and $\epsilon$ corresponding to InAs. In particular, the effective-mass value seems to depend on whether it is extracted from capacitance of from FIR spectroscopy. We have checked that if we take ${ }^{14,16} m^{*}=0.08$ we achieve a better description of the "dot" peaks in Figs. 3 and 6 at the price of spoiling the description of diamond and triangle peaks. Yet, the pattern looks qualitatively similar.

Finally, we show in Fig. 8 the addition energies of both rings as compared with the gate voltage shift of the lowest capacitance maximum. ${ }^{2,22}$ It can be seen that the agreement between theory and experiment is rather poor. At $B \sim 12 \mathrm{~T}$ the calculations underestimate the shift voltage around a factor of 3 for $N=2$, and of 2 for $N=1$. We recall that the agreement between capacitance spectroscopy experiments and exact-diagonalization calculations of few electron quantum dots is also only qualitative. ${ }^{24,25} \mathrm{We}$ cannot discard that using a different radius $R_{0}$ for each ring would not improve 
the agreement but again, we have not tried this possibility to avoid too much parameter fitting in the calculation. The electron-electron interaction determines the energy difference between $\mu(1)$ and $\mu(2)$ at $B=0$. A small bump in $\mu(2)$ at $B \sim 2-3 \mathrm{~T}$ is the signature of full polarization. A similar structure shows up in the experimental points but between 3 and $4 \mathrm{~T}$. Interestingly also, the change in the elec- tronic structure at $B \sim 8 \mathrm{~T}$ is visible in the calculated addition energy $\mu(2)$.

This work has been performed under Grant Nos. PB981247 from DGESIC, Spain, and 1998SGR00011 from Generalitat of Catalunya. A.E. acknowledges support from the DGESIC (Spain), and A.L. from the German Ministry of Science (BMBF).
${ }^{1}$ A. Lorke and R. J. Luyken, Physica B 256, 424 (1998).

${ }^{2}$ A. Lorke, R. J. Luyken, A. O. Govorov, J. P. Kotthaus, J. M. Garcia, and P. M. Petroff, Phys. Rev. Lett. 84, 2223 (2000).

${ }^{3}$ C. Dahl, J. P. Kotthaus, H. Nickel, and W. Schlapp, Phys. Rev. B 48, 15480 (1993).

${ }^{4}$ C. R. Proetto, Phys. Rev. B 46, 16174 (1992).

${ }^{5}$ E. Zaremba, Phys. Rev. B 53, R10 512 (1996).

${ }^{6}$ T. Chakraborty and P. Pietiläinen, Phys. Rev. B 50, 8460 (1994).

${ }^{7}$ V. Gudmundsson and Á. S. Loftsdóttir, Phys. Rev. B 50, 17433 (1994).

${ }^{8}$ L. Wendler, V. M. Fomin, A. V. Chaplik, and A. O. Govorov, Phys. Rev. B 54, 4794 (1996).

${ }^{9}$ V. Halonen, P. Pietiläinen, and T. Chakraborty, Europhys. Lett. 33, 377 (1996).

${ }^{10}$ K. Niemelä, P. Pietiläinen, P. Hyvönen, and T. Chakraborty, Europhys. Lett. 36, 533 (1996).

${ }^{11}$ W.-C. Tan and J. C. Inkson, Phys. Rev. B 60, 5626 (1999).

${ }^{12}$ A. Emperador, M. Barranco, E. Lipparini, M. Pi, and Ll. Serra, Phys. Rev. B 59, 15301 (1999).

${ }^{13}$ I. Magúsdóttir and V. Gudmundsson, cond-mat/9907216 (unpublished).

${ }^{14}$ M. Fricke, A. Lorke, J. P. Kotthaus, G. Medeiros-Ribeiro, and P. M. Petroff, Europhys. Lett. 36, 197 (1996).

${ }^{15}$ A. Lorke, M. Fricke, B. T. Miller, M. Haslinger, J. P. Kotthaus, G. Medeiros-Ribeiro, and P. M. Petroff (unpublished); Inst.
Phys. Conf. Ser. 155, 803 (1997).

${ }^{16}$ B. T. Miller, W. Hansen, S. Manus, R. J. Luyken, A. Lorke, J. P. Kotthaus, S. Muant, G. Medeiros-Ribeiro, and P. M. Petroff, Phys. Rev. B 56, 6764 (1997).

${ }^{17}$ M. Ferconi and G. Vignale, Phys. Rev. B 50, 14722 (1994).

${ }^{18}$ M. Pi, M. Barranco, A. Emperador, E. Lipparini, and Ll. Serra, Phys. Rev. B 57, 14783 (1998).

${ }^{19}$ L1. Serra, M. Barranco, A. Emperador, M. Pi, and E. Lipparini, Phys. Rev. B 59, 15290 (1999).

${ }^{20}$ F. Pederiva, C. J. Umrigar, and E. Lipparini, Phys. Rev. B (to be published).

${ }^{21}$ We have adopted the convention that $l$ represents the SP orbital angular momentum change of sign (Ref. 17). The applied $B$ points towards the positive direction of the $z$ axis. Consequently, most occupied $S P$ levels have a negative orbital angular momentum, the total orbital angular momentum $L_{z}$ is zero or negative, and the total spin $S_{z}$ is zero or positive.

${ }^{22}$ The data for the $N=1$ ring were not given in Ref. 2 and are presented here.

${ }^{23}$ K. Bollweg, T. Kurth, D. Heitmann, E. Vasiliadou, K. Eberl, and H. Brugger, Phys. Rev. B 52, 8379 (1995).

${ }^{24}$ R. C. Ashoori, H. L. Stormer, J. S. Weiner, L. N. Pfeiffer, K. W. Baldwin, and K. W. West, Phys. Rev. Lett. 71, 613 (1993).

${ }^{25}$ J. J. Palacios, L. Martin-Moreno, G. Chiappe, E. Louis, and C. Tejedor, Phys. Rev. B 50, 5760 (1994). 